\title{
Analysis of Thermal Limitations in High-Speed Microcavity Saturable Absorber All-Optical Switching Gates
}

\author{
David Massoubre, Jean-Louis Oudar, Arthur O'Hare, Mathilde Gay, Laurent Bramerie, \\ Jean-Claude Simon, Alexandre Shen, and Jean Decobert
}

\begin{abstract}
The limitations owing to device heating and thermooptic effects in high-speed quantum-well microcavity saturable absorber devices are investigated both theoretically and experimentally. A simplified theoretical description of the device electronic, thermal, and optical properties is developed and applied to the modeling of the device switching characteristics for reamplification + reshaping step $(2 \mathrm{R})$ all-optical regeneration. These predictions are compared to nonlinear optical measurements performed with switching pulses of fixed duration and variable repetition rate on two devices with significantly different thermal properties. It is shown that proper optimization of the device thermal properties is crucial to avoid the degradation of device performance at high bit rate. It is also shown that the negative effects of optically induced heating on the switching contrast may be compensated to some extent by operating the device on the long wavelength side of the microcavity resonance.
\end{abstract}

Index Terms-All-optical switching gate, asymmetric FabryPerot devices, high-speed optical techniques, nonlinear optics, optical regeneration, optical saturable absorption, semiconductor quantum well, thermal effects.

\section{INTRODUCTION}

$\mathbf{T}$ HE RAPID traffic growth in optical transmissions indicates that future optical networks will need to operate at $160 \mathrm{~Gb} / \mathrm{s} / \mathrm{channel}$. In such systems, ultrahigh-speed regenerators will be required to combat cumulative transmission impairments. Because of the speed limitations of optoelectronic devices and of their increasing complexity with increasing bit rate, all-optical devices are very attractive for low-cost inline signal processing [1]. These devices need a nonlinear intensity transfer function (output power versus input power) to enable them to perform their nonlinear gate role. Different nonlinear optical gates have been realized in various ways such as fiber-based nonlinear optical loop mirror (NOLM) [2],

Manuscript received February 9, 2006; revised May 29, 2006. This work was supported in part by the French Telecom Research Network (RNRT-ASTERIX project), the Conseil Général de l'Essonne, and the Ministere de l'Industrie.

D. Massoubre and J.-L. Oudar are with the Laboratory for Photonics and Nanostructures, Centre National de la Recherche Scientifique UPR20, 91460 Marcoussis, France (e-mail: david.massoubre@lpn.cnrs.fr).

A. O'Hare is with the School of Physics, Dublin Institute of Technology, Dublin, Ireland.

M. Gay, L. Bramerie, and J.-C. Simon are with the Laboratoire d'Optronique, Centre National de la Recherche Scientifique UMR FOTON 6082, ENSSAT/ Université de Rennes1, 22300 Lannion, France.

A. Shen and J. Decobert are with Alcatel-Thales III-V Lab, 91460 Marcoussis, France.

Digital Object Identifier 10.1109/JLT.2006.879502 semiconductor optical amplifier (SOA)-based interferometers [3], electro-absorption modulators [4], passive saturable absorbers (SA) [5], and bistable lasers [6]. These nonlinear gates work either by cross-regeneration, where data are transferred from one wavelength to another (wavelength conversion), or by self-regeneration, where data are self-modulated. The latter approach is simpler to implement because it does not require an external laser source and does not introduce a wavelength shift. Among these nonlinear gates, SA-based gates have generated considerable interest in all-optical switching owing to their large nonlinear optical effect, small size, and fully passive operating mode (no bias voltage, no Peltier cooler), thus offering a potentially low-cost solution to signal regeneration [7]. InP-based multiple-quantum-well SAs (MQW-SAs) are currently being investigated for optical telecommunication signal processing because of the facility to obtain an absorption peak at $1.55 \mu \mathrm{m}$ [5], [7]-[10]; recently, however, GaAs-based MQWSA [11] and carbon-nanotube-based SA [12] have also been investigated.

To be used as low-cost regenerators at high bit rate, MQWSA-based gates must combine numerous characteristics such as a switching energy and insertion loss as low as possible, a contrast ratio near $5 \mathrm{~dB}$ [13], and an ultrafast recovery time (typically $1.5 \mathrm{ps}$ for $160-\mathrm{Gb} / \mathrm{s}$ transmission lines). They need to be polarization insensitive, easy to make, and compatible with wavelength-division-multiplexed (WDM) systems. To amplify the nonlinear response, the SA structure is inserted inside an asymmetric Fabry-Perot microcavity and operates at resonance. This microcavity, which is designed to obtain a low reflectance at low incident energy, enhances the intracavity electromagnetic field and thus, combined with the excitonic absorption, augments the nonlinearity and decreases the effective switching energy [14]. Furthermore, it is to be used at normal incidence, thus yielding intrinsic polarization-insensitive operation. MQW-SAs typically have a rather long recovery time (in the nanosecond range). In order to significantly decrease the response time of these materials and to make them compatible with high bit rate operation, capture and recombination centers must be introduced during or after crystal growth by means of low-temperature molecular beam epitaxy [15], ion implantation [16], heavy ion irradiation [17], or Be or Fe doping [18], [19]. These methods of damage creation have been reported to provide recovery times in the sub-picosecond range [20], [21]. 


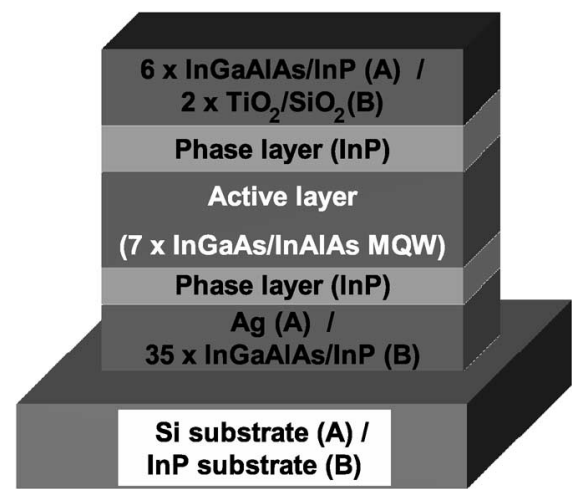

Fig. 1. Schematic device structure of structures A and B.

Such a device based on the combination of heavy-ion irradiation with a compact microcavity has been used as part of a reamplification and reshaping step $(2 \mathrm{R})$ regenerator in $40-\mathrm{Gb} / \mathrm{s}$ long-haul transmission lines and has allowed considerable improvement of the propagation distance [22]. Furthermore, a tunability of over $13 \mathrm{~nm}$ has been shown with a $10-\mathrm{Gb} / \mathrm{s}$ recirculating loop [23], and an extinction ratio enhancement of up to $6 \mathrm{~dB}$ has been achieved at $160 \mathrm{GHz}$ within a wavelength range of $8 \mathrm{~nm}$ [24].

To extend the functionality of these devices toward higher bit rates, such as $160 \mathrm{~Gb} / \mathrm{s}$, it is important to investigate their thermo-optic properties. Indeed, owing to the dissipative nature of SA optical switches, the main challenge is to obtain not only a fast response time but also robustness with respect to the operating power of high-repetition-rate optical systems.

Increasing the response speed of the device is usually done at the expense of an increased saturation power, which tends to raise the temperature of the device active region. It will be shown that the most direct effect of this optically induced temperature rise is the shift of the microcavity resonance toward longer wavelengths (red shift). An additional effect is the wellknown bandgap shift with temperature-it is also a red shift, and its importance depends on the relative positions of the cavity and the exciton resonances. To minimize these effects, it is important to maintain a good heat dissipation capability in the device structure. In this paper, we investigate the thermal limitations in high-speed quantum-well microcavity SA devices. A detailed modeling of the switching characteristics, in the presence of thermal effects, was developed and compared to nonlinear optical measurements performed with switching pulses of fixed duration and variable repetition rate on two devices with significantly different thermal properties.

\section{Device FAbrication}

In this paper, two devices have been used and are referred as structure A and structure B. The device structures are schematized in Fig. 1 and are similar to those reported elsewhere [22], [24]. The active layers consist of $7 \times$ InGaAs/InAlAs MQW for the two structures, grown by metal-organic vapor phase epitaxy (MOVPE) upon an InP substrate, and are suitably located at the antinode of the intracavity intensity inside a Fabry-Perot microcavity. As the InGaAs/InAlAs MQW absorption is polarization insensitive, there is no absorption dichroism
[25]-[27]. After MOVPE growth, the structures underwent $11 \mathrm{MeV} \mathrm{Ni}{ }^{6+}$ ion irradiation at a dose of $1 \times 10^{12} \mathrm{~cm}^{-2}$ (structure A) or $4 \times 10^{11} \mathrm{~cm}^{-2}$ (structure B). Such energetic ions create clusters of defects along the ion path through the active layers. For structure A, the bottom and top mirrors were, respectively, made by deposition of a thin silver ( $\mathrm{Ag}$ ) layer and by a $6 \times \mathrm{InP} / \mathrm{InGaAsP}$ distributed Bragg reflector (DBR) deposited during the MOVPE. To fabricate structure A, a topdown mounting was necessary. This was done by bonding the $\mathrm{Ag}$ film onto a silicon wafer by an indium-gold isothermal solid-liquid interdiffusion technique [28], which facilitates heat dissipation. In the case of structure $\mathrm{B}$, the bottom and top mirrors are, respectively, made of a $35 \times \mathrm{InP} / \mathrm{InGaAlAs}$ DBR deposited during the MOVPE growth and of a two-period dielectric coating $\left(2 \times \mathrm{SiO}_{2} / \mathrm{TiO}_{2}: \lambda / 4 \lambda / 4\right)$. To enhance the nonlinear response, the cavities were designed to have a minimum reflectivity close to zero at low intensity (impedance matching) and to be wavelength matched to the quantum-well exciton absorption line. The device switching times $\tau_{r}$ have been measured with a classical pump and probe setup that used 1.2-ps pulses from a passive mode-locked erbium-doped fiber laser operating at $1550-\mathrm{nm}$ wavelength. The results showed a $1 / e$ recovery time $\tau_{r}$ of $1.5 \mathrm{ps}$ for structure A and of 3 ps for structure B. These values are short enough to work up to 160 and $40 \mathrm{~Gb} / \mathrm{s}$, respectively.

\section{Device Modeling}

The numerical computation performs a self-consistent resolution of the variations of carrier density $N_{c}$ and active layer temperature $T_{\text {act }}$ with the parameters characterizing the incident optical signals at a given laser wavelength $\lambda_{\text {work }}$. In spite of the complexity of these phenomena, the calculations are essentially the solution of a series of steady-state equations. Some key features of the modeling are the following: 1) A Fabry-Perot model is used to calculate both the intracavity power resonance behavior and the spectral dependence of the device reflectance $R$. 2) A microscopic model of the quantumwell absorption describes its dependence on $\lambda_{\text {work }}, N_{c}$, and $T_{\text {act }}$. 3) It includes a phenomenological description of the resonance spectral shift with $N_{c}$ and $T_{\text {act }}$ based on independently measured data. 4) The evaluation of the absorbed power $P_{\text {abs }}$ takes into account the saturable loss owing to the quantum-well absorption (giving a dissipated power proportional to $N_{c}$ ) and an additional nonsaturable loss essentially owing to the bottom mirror loss (proportional to the intracavity power). These different phenomena and associated models are described below.

\section{A. Device Time Scales}

Owing to the very large difference in time scales between the absorption saturation itself and the thermal effects, they can be discussed and evaluated separately. The time scale for absorption saturation in QWs is related to the carrier recombination time $\tau_{r}$, which in this study is shortened by the introduction of recombination centers created by means of ion irradiation. For adequate response to digital optical signals, the absorption saturation will have to process consecutive pulses 
independently. $\tau_{r}$ must then be (typically four times) shorter than the bit duration $\tau_{\mathrm{bit}}$, and $\tau_{\mathrm{bit}}=1 / B_{r}$, where $B_{r}$ is the bit rate. On the other hand, the time scale of thermal effects is of the order of microseconds, so an optically induced steady-state absorber temperature rise can be considered. This may reasonably be assumed to be proportional to the average absorbed power.

\section{B. Fabry-Perot Model}

The microcavity reflectivity $R$ is readily calculated from the usual procedure of Airy summation in a Fabry-Perot cavity [29]. For the simple case of normal incidence, it can be expressed as

$$
R=\frac{\left(r_{b a}-r_{f}\right)^{2}+4 r_{f} r_{b a} \sin ^{2} \phi}{\left(1-r_{f} r_{b a}\right)^{2}+4 r_{f} r_{b a} \sin ^{2} \phi}
$$

where $r_{b a}=\sqrt{R_{b}} e^{\left(-\eta N_{\mathrm{qw}} \Gamma\right)}$ and $r_{f}=\sqrt{R_{f}}$.

In these equations, $R_{f}$ and $R_{b}$ are, respectively, the front and back mirror reflectances, $\eta$ is the single-pass absorption per QW, $N_{\mathrm{qw}}$ is the number of QW, $\Gamma$ is the longitudinal confinement factor of the microcavity [30], and $\phi$ is the singlepass dephasing. $R$ is dependent on the working wavelength $\lambda_{\text {work }}$ and on the carrier density $N_{c}$ through the parameters $\eta$ and $\phi$. To reduce the computation time, this dependence was described through a phenomenological model based on independently measured data and on simulations of the nonlinear absorption obtained from the equation of Bethe-Salpeter in momentum space [31], [32]. The numerical results of $\eta=f\left(\lambda_{\text {work }}, N_{c}\right)$ obtained with this model and shown in Fig. 2(a) are similar to experimental results reported elsewhere [33], [34]. $\phi=f\left(\lambda_{\text {work }}, N_{c}\right)$ is given by

$$
\phi=\left(\lambda_{\text {work }}-\lambda_{\text {res }}\right)\left(\frac{d \phi}{d \lambda}\right)_{\lambda_{\text {res }}}+\frac{d \phi}{d N_{c}} N_{c}
$$

where $\lambda_{\text {res }}$ is the microcavity resonance wavelength, and $d \phi / d \lambda$ and $d \phi / d N_{c}$ are obtained from experimental results. Fig. 2(b) shows the optical reflectivity spectra calculated for structure A with this model at different values of $N_{c}$. Experimental spectra measured for this structure at different incident energies $E_{\text {in }}$ are also displayed for comparison. The experimental data have been measured with a mode-locked source producing picosecond pulses at $23 \mathrm{MHz}$ (this low repetition rate prevents thermal effects). The good agreement observed between the phenomenological model and the experimental results serves to validate the modeling of the microcavity. As expected, when increasing $N_{c}$ or $E_{\text {in }}$, the quantum-well absorption decreases, and the microcavity reflectivity increases. Furthermore, the resonance wavelength slightly shifts toward shorter wavelengths (blue shift).

The photoexcited carrier density dynamics of the quantumwell SA is described by the rate equation

$$
\frac{d N_{c}}{d t}=\frac{\eta I_{\mathrm{qw}}}{\hbar \omega}-\frac{N_{c}}{\tau_{r}}
$$

where $I_{\mathrm{qw}}$ is the effective intensity within the active layers, $\tau_{r}$ is the carrier recombination time, $\hbar$ is the reduced Planck's

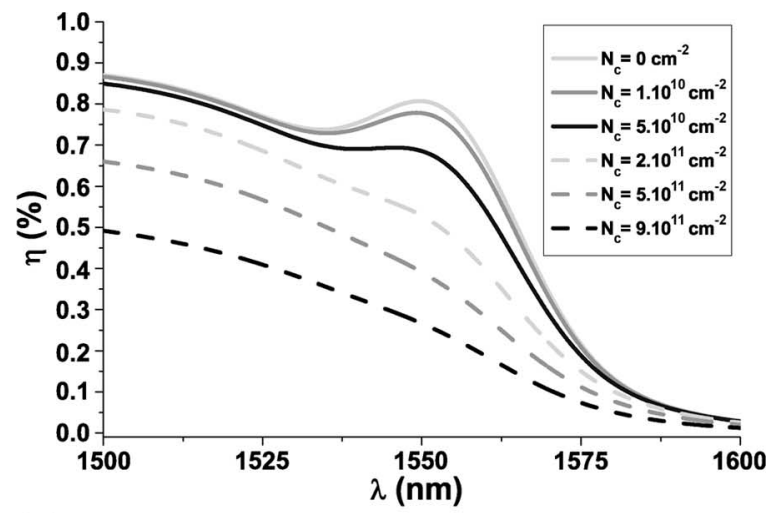

(a)

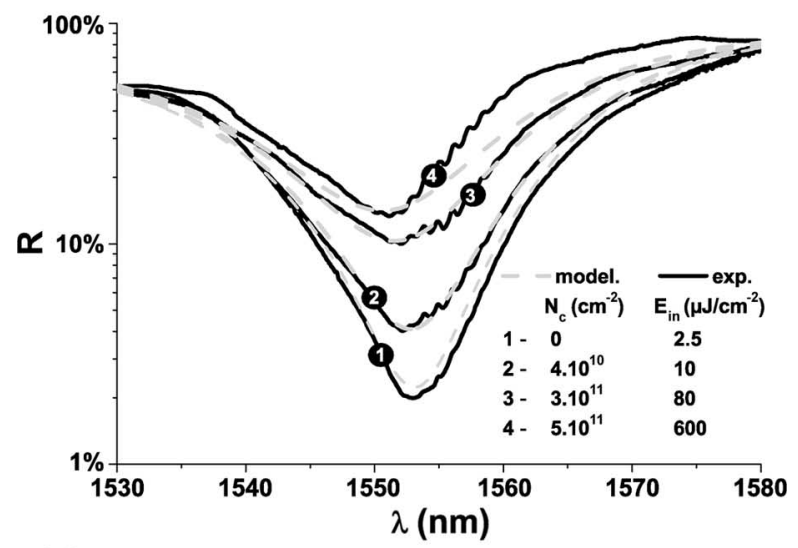

(b)

Fig. 2. (a) Nonlinear optical absorption spectra in InGaAs-based quantum wells calculated from the phenomenological model for different carrier densities $N_{c}$. The excitonic absorption central wavelength $\lambda_{x}$ is set to $1555 \mathrm{~nm}$. (b) Numerical and experimental optical reflectivity spectra of structure A for different values of $N_{c}$ and of incident energy $E_{\text {in }}$, respectively. $d \phi / d \lambda$ and $d \phi / d N_{c}$ are set to $0.006 \mathrm{rad} / \mathrm{nm}$ and $2.10^{-14} \mathrm{rad} \cdot \mathrm{cm}^{2}$, respectively. Modeling and measurements have been done at $300 \mathrm{~K}$ and with $\lambda_{\text {res }}=1553 \mathrm{~nm}$.

constant, and $\omega$ is the incident laser angular frequency. With the steady state condition $d N_{c} / d t=0$ inserted into (3), the expression for the local carrier density $N_{c}$ is obtained from

$$
N_{c}=\eta \frac{\tau_{r}}{\hbar \omega} I_{\mathrm{qw}} .
$$

$I_{\mathrm{qw}}$ is related to the maximal intracavity intensity $I_{\max }$ through [30]

$$
I_{\mathrm{qw}}=\frac{\Gamma}{2} I_{\max }
$$

where $I_{\max }$ is related to the incident intensity $I_{0}$ at normal incidence through

$$
I_{\max }=\frac{\left(1+r_{b a}\right)^{2}\left(1-r_{f}^{2}\right)}{\left|1-r_{b a} r_{f} e^{(2 i \phi)}\right|^{2}} I_{0} .
$$

Inserting (6) into (5), and with $r_{b a}$ close to 1, (4) becomes

$$
N_{c}=\frac{\Gamma\left(1-R_{f}\right) \eta\left(N_{c}\right)}{\left(1-r_{b a} r_{f}\right)^{2}+4 r_{b a} r_{f} \sin ^{2} \phi} \frac{2 \tau_{r}}{\hbar \omega} I_{0} .
$$




\section{Thermal Dependence}

One may note that the thermal effects have been neglected until now. Indeed, at low pulse repetition rate, the ratio of average power $P_{\text {ave }}$ to peak power $P_{\text {peak }}$ is very small, so the absorption saturation, which depends directly on the peak power, is obtained with a low $P_{\text {ave }}$ [cf. Fig. 2(b)]. At high pulse repetition rate, however, this ratio increases, and a much higher $P_{\text {ave }}$ is necessary to saturate the absorption. Thus, thermal effects have to be taken into account to model the device behavior.

The rise of the active layer temperature $T_{\text {act }}$ shifts the direct bandgap $E_{G}$ following the empirical Varshni equation [35]. Above $200 \mathrm{~K}$, this shift is practically linear with temperature and can be approximated by

$$
E_{G}\left(T_{\mathrm{act}}\right)=E_{G}(300)+\frac{d E_{G}}{d T_{\mathrm{act}}}\left(T_{\mathrm{act}}-300\right) .
$$

For the InGaAs lattice matched to InP, $d E_{G} / d T_{\text {act }}$ is equal to $-0.34 \mathrm{meV} \cdot \mathrm{K}^{-1}$ [36], [37]. This temperature dependence of $E_{G}$ induces a relative translation of the absorption spectrum of $0.04 \% / \mathrm{K}$, i.e., of $\sim 0.65 \mathrm{~nm} / \mathrm{K}$ at $1550 \mathrm{~nm}$, toward longer wavelengths (red shift) if $T_{\text {act }}$ increases. The microcavity refractive index and the microcavity thickness also depend on temperature and produce, with increasing temperature, a linear red shift of the cavity mode with temperature. This linear shift is given by

$$
\lambda_{\text {res }}\left(T_{\text {act }}\right)=\lambda_{\text {res }}(300)+\frac{d \lambda_{\text {res }}}{d T_{\text {act }}}\left(T_{\text {act }}-300\right) .
$$

Our experimental data gave a value of $0.09 \mathrm{~nm} / \mathrm{K}$ for $d \lambda_{\text {res }} / d T_{\text {act. }}$. This value is in good agreement with values reported elsewhere [38], [39].

The average temperature $T_{\text {act }}$ of the active zone is calculated from the average absorbed power $P_{\text {abs }}$ through

$$
T_{\text {act }}=T_{s}+R_{\mathrm{th}} P_{\mathrm{abs}}\left(N_{c}\right)
$$

where $T_{s}$ is the substrate temperature, which is typically $300 \mathrm{~K}$, and $R_{\mathrm{th}}$ is the overall effective thermal resistance of the microcavity, which is determined from experimental data (see Section IV-B).

The evaluation of $P_{\mathrm{abs}}$ takes into account the saturable loss owing to the quantum-well absorption and an additional nonsaturable loss essentially owing to the bottom mirror loss. Assuming a Gaussian spatial profile input pulse and, consequently, a Gaussian carrier distribution, the average power absorbed by the active layers is calculated from

$$
P_{\mathrm{qw}}=\rho \frac{\pi \omega_{o}^{2}}{8} N_{\mathrm{qw}} \frac{\hbar \omega}{\tau_{r}} N_{c}
$$

where $\omega_{o}$ is the spot diameter at $1 / e^{2}$ intensity. In (11), a filling factor (FF) $\rho$ has been introduced to account for the ratio $P_{\text {ave }} / P_{\text {peak }}$. For pulses of duration $\tau_{\text {pulse }}$ (full-width at half-maximum, FWHM) and repetition rate $f_{r}$, one has $\rho=$ $\tau_{\text {pulse }} f_{r}$.
The power $P_{b}$ dissipated by the nonsaturable loss is proportional to the incident intracavity intensity $I_{b}$ on the back mirror and is expressed as

$$
P_{b}=\rho \frac{\pi \omega_{o}^{2}}{8}\left(1-R_{b}\right) I_{b} .
$$

Furthermore, $I_{b}$ is related to the average intensity $I_{\mathrm{qw}}$ within the active layers according to

$$
I_{\mathrm{qw}}=2 \Gamma I_{b} .
$$

Combining (11)-(13) and (4), we can calculate the total absorbed power $P_{\mathrm{abs}}$ as a function of $N_{c}$ through

$$
\begin{aligned}
P_{\mathrm{abs}}\left(N_{c}\right) & =P_{\mathrm{qw}}+P_{b} \\
& =\rho \frac{\pi \omega_{o}^{2}}{8} \frac{\hbar \omega}{\tau_{r}}\left[N_{\mathrm{qw}}+\frac{\left(1-R_{b}\right)}{2 \Gamma \eta\left(N_{c}\right)}\right] N_{c} .
\end{aligned}
$$

From this model, it is found that the optically induced temperature rise is essentially proportional to the thermal resistance $R_{\mathrm{th}}$, the signal FF $\rho$, and the total volume of illuminated quantum wells, and is inversely proportional to the carrier lifetime $\tau_{r}$.

\section{Results And Discussion}

\section{A. Experimental Setup}

To provide an experimental test of this model, the evolution of the device switching characteristics was measured under high average power input signals with the setup described in Fig. 3. In this setup (of the wavelength conversion type), a modulated pump beam and a continuous wave $(\mathrm{CW})$ probe beam were focused on the device with a fiber-pigtailed highaperture lens providing a focused spot $\omega_{o}$ of 5- $\mu$ m diameter at $1 / e^{2}$ intensity. After spectral filtering and optical amplification, the extinction ratio of the reflected probe signal was measured by a fast photodetector and oscilloscope combination. The pump signal consists of a repeated digital optical word of duration $N \times \tau_{\text {bit }}$ [one bit "1" followed by $(N-1)$ bit " 0 "], where $N$ is a varying integer, and $\tau_{\text {bit }}$ is the bit duration. Each word contains one return-to-zero (RZ) optical pulse of FWHM duration $\tau_{\text {pulse. }}$. The FF is calculated as the ratio of pulse length to word length and can be varied simply by varying $N$, i.e.,

$$
F F=\frac{\tau_{\text {pulse }}}{\tau_{\text {bit }}} \frac{1}{N}=\frac{P_{\text {ave }}}{P_{\text {peak }}}=\tau_{\text {pulse }} B_{r}=\rho .
$$

Since energy is supplied only during the optical pulse, FF also gives the ratio of average power to peak power and is therefore equal to $\rho$, as defined in Section III.

In this experiment, the pump signal was produced by a $10-\mathrm{Gb} / \mathrm{s} \mathrm{RZ}$ optical word generator having $\tau_{\mathrm{bit}}=100 \mathrm{ps}$ and pulse duration $\tau_{\text {pulse }}=50 \mathrm{ps}$.

\section{B. Thermal Resistance}

To compute the numerical results, the microcavity effective thermal resistance must be known. Although the two 


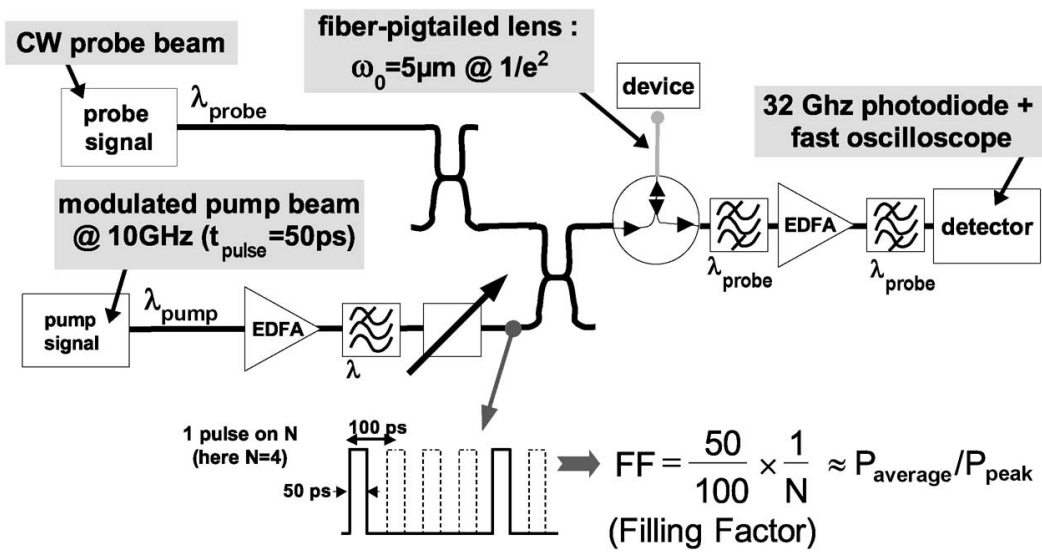

Fig. 3. Experimental setup and definition of the FF. $P_{\text {ave }}$ and $P_{\text {peak }}$ are the average and peak power, respectively.

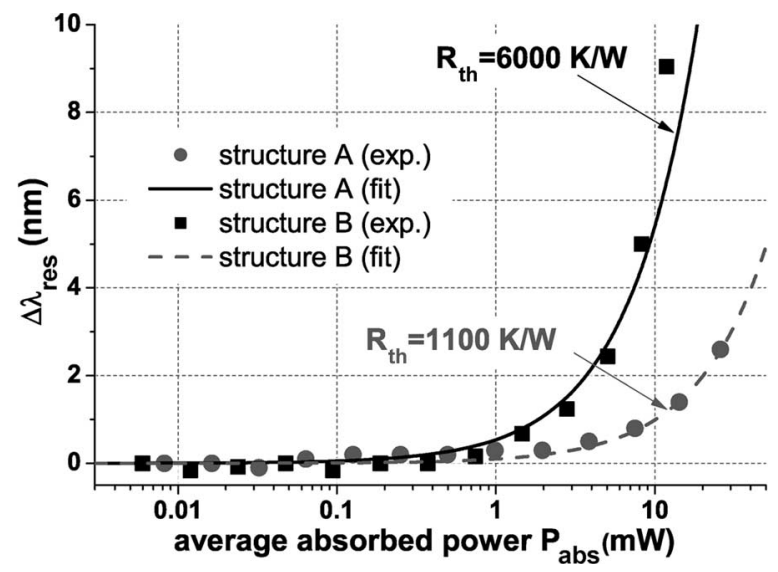

Fig. 4. Experimental values (symbol) of the resonance wavelength shift $\Delta \lambda_{\text {res }}$ versus the average absorbed power $P_{\text {abs }}$ measured on structures $\mathrm{A}$ and B. The fitting curves follow the combination of (9) and (10).

microcavities investigated here are similar, they are made with different materials, so their thermal resistances differ from each other, leading to different thermal behaviors. This is illustrated in Fig. 4, where the resonance wavelength red shift $\Delta \lambda_{\text {res }}$ is plotted versus the average absorbed power $P_{\text {abs }}$ for the two microcavities. $\Delta \lambda_{\text {res }}$ was measured with an amplified spontaneous emission (ASE) source produced by an optical amplifier [erbium-doped fiber amplifier (EDFA)]. The reflected input beam was detected by an optical spectrum analyzer (OSA).

It appears that the resonance wavelength from structure B shifts much more quickly than the one from structure A. This means that structure B heats much more than structure A because of a higher effective thermal resistance. The experimental data have been fitted with a linear function, and knowing the experimental value of $d \lambda_{\text {res }} / d T_{\text {act }}(=0.09 \mathrm{~nm} / \mathrm{K}$ [38], [39]), $R_{\text {th }}$ is readily deduced. The fitting curves give $R_{\text {th }}$ values of 1100 and $6000 \mathrm{~K} / \mathrm{W}$ for structures $\mathrm{A}$ and $\mathrm{B}$, respectively. This significant difference in $R_{\mathrm{th}}$ values comes mainly from the materials used to make the microcavities' back mirrors. The back mirror of structure B is made with semiconductor materials known to have poor thermal conductance $(0.68$ and $0.045 \mathrm{~W} / \mathrm{K} \cdot \mathrm{cm}$ for InP and InGaAlAs, respectively, at room temperature), while in structure $\mathrm{A}$, the back mirror is made with a thin silver layer that is a very good thermal conductor
$(4.27 \mathrm{~W} / \mathrm{K} \cdot \mathrm{cm}$ at room temperature). Furthermore, structure A is transferred on a silicon wafer thanks to a metallic bonding layer, both of them having a good thermal conductance. As a result, structure A has better thermal conductance than structure B. Independent calculations of the thermal resistance of these structures were performed by assuming a Gaussian profile for both carrier distribution and absorbed intensity and an infinite lateral extent absorber layer. These independent thermal modeling gave thermal resistances of 1350 and $5600 \mathrm{~K} / \mathrm{W}$, respectively, for the structures $\mathrm{A}$ and $\mathrm{B}$, which are in good agreement with the fitted values.

\section{Experimental and Numerical Switching Characteristics}

In order to clearly demonstrate the effects of optically induced heating on the device switching characteristics, we compared the performances of two devices (one with structure B and one with structure A), which have been shown to have markedly different thermal behavior.

1) The first experiment was performed with a structure $B$ device for two different values of FF (0.25 and 0.1). The pump wavelength was set to $1549 \mathrm{~nm}$, and the probe wavelength was adjusted to the resonance wavelength $\left(\lambda_{\text {work }} \sim 1557 \mathrm{~nm}\right)$. Fig. 5(a) displays the contrast $C$ as a function of average incident power $P_{\text {ave }} . C$ is the logarithmic ratio of the device reflectance $R_{\text {on }}$ when illuminated by a bit " 1 " to $R_{\text {off }}$ the device reflectance when illuminated by a bit " 0 " $\left(P_{\text {peak }}=0 \mathrm{~mW}\right)$, i.e., $C=$ $10 \log \left(R_{\text {on }} / R_{\text {off }}\right)$. Numerical calculations have also been done, and the parameter values used for these simulations are indicated in Table I. The back mirror reflectance was set to take into account the residual absorption owing to the semiconductor DBR ion irradiation [40]. Indeed, as the irradiation step is a post-growth process, the allsemiconductor Bragg mirror undergoes an ion implantation leading to higher insertion loss.

A very good agreement is observed between the numerical and the experimental results. Fig. 5(a) shows, at first, that $C$ is strongly dependent on $\mathrm{FF}$, but whatever the value of $\mathrm{FF}$, the curves present the same trend, i.e., an increase with incident power of up to $10 \mathrm{dBm}$ and then a quick decrease. To explain these results, the reflectance 


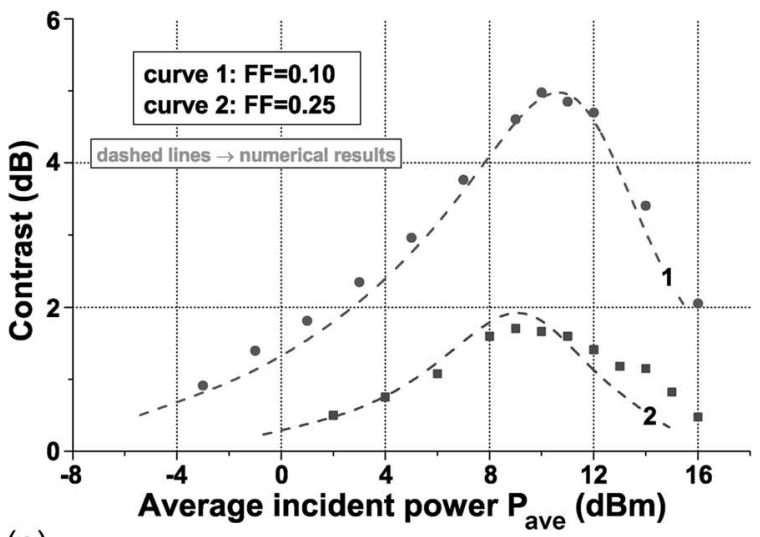

(a)

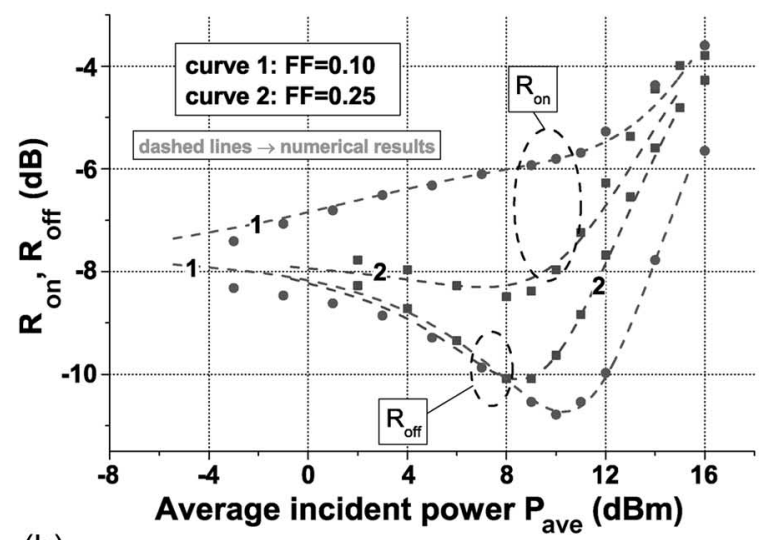

(b)

Fig. 5. Experimental (symbols) and numerical (full curve) results from structure B for (a) switching contrast $C$ and (b) device reflectances $R_{\text {on }}$ and $R_{\text {off }}$ when illuminated by bit " 1 " and " 0 ," respectively, versus the average incident power $P_{\text {ave }}$ and obtained for two values of FF (0.25 and 0.1$)$. Numerical calculation and experiment are done with an initial active layer temperature $T_{\text {act }}$ of $300 \mathrm{~K}$ and with $\lambda_{\text {work }}$ tuned to $\lambda_{\text {res }}$ at low incident average power.

TABLE I

PARAMETER VALUeS USED FOR THE SimUlations ON STRUCTURES A AND B

\begin{tabular}{ccc}
\hline \hline Parameters & structure A & structure B \\
\hline$\tau_{r}(\mathrm{ps})$ & 1.5 & 3 \\
$\mathrm{R}_{b}$ & 0.95 & 0.95 \\
$\mathrm{R}_{f}$ & 0.71 & 0.805 \\
$\mathrm{~N}_{q w}$ & 7 & 7 \\
$\Gamma$ & 1.64 & 1.64 \\
$\mathrm{R}_{t h}\left(\mathrm{~K} . \mathrm{W}^{-1}\right)$ & 1100 & 6000 \\
$\mathrm{~d} \phi / \mathrm{d} \lambda(\mathrm{rad} / \mathrm{nm})$ & 0.006 & 0.015 \\
$\mathrm{~d} \phi / \mathrm{dN} N_{c}\left(\mathrm{rad} . \mathrm{cm}^{2}\right)$ & $2.10^{-14}$ & $2.310^{-14}$ \\
$\lambda_{X}(\mathrm{~nm})$ & 1549 & 1539 \\
$\lambda_{r e s}(\mathrm{~nm})$ & 1557 & 1557.2 \\
$\lambda_{\text {work }}(\mathrm{nm})$ & 1560.5 & 1557 \\
$\mathrm{~T}_{\text {act }}(\mathrm{K})$ & 300 & 300 \\
$\omega_{o}(\mu \mathrm{m})$ & 5 & 5 \\
\hline \hline
\end{tabular}

values $R_{\mathrm{on}}$ and $R_{\mathrm{off}}$ from the same structure have been plotted versus the average incident power in Fig. 5(b). This plot shows that the behaviors of both $R_{\text {on }}$ and $R_{\text {off }}$ as a function of the incident power and the FF are totally different. On one hand, $R_{\text {on }}$ continuously increases with $P_{\text {ave }}$. This arises because $R_{\text {on }}$ depends mainly on the absorption, which decreases with the peak power, hence giving a higher reflectance [cf. Fig. 2(a) and (b)]. Furthermore, for a given value of $P_{\text {ave }}, R_{\text {on }}$ decreases with increasing FF because the peak power becomes lower as described by (15). On the other hand, $R_{\text {off }}$ is seen to slowly decrease and then to strongly increase above a given incident power (typically $9 \mathrm{dBm}$ ). This is essentially owing to the heating effects of the optical signal that cause both the cavity and exciton resonances to shift to longer wavelengths with increasing temperature. For low incident power, and because $d \lambda_{x} / d T_{\text {act }} \gg$ $d \lambda_{\text {res }} / d T_{\text {act }}$, the exciton wavelength red shift enhances the impedance matching leading to a smaller value of $R_{\text {off }}$, but for an incident $P_{\text {ave }}$ higher than $\sim 9 \mathrm{dBm}$, the cavity resonance red shift becomes more important and leads to a decrease of the switching contrast. As $\lambda_{\text {res }}$ is detuned from the signal wavelength, $R_{\text {off }}$ automatically increases and compensates the (slower) increase of $R_{\mathrm{on}}$, leading to a decrease of the switching contrast. As these heating effects are mainly owing to the average incident power, they are relatively independent of FF and therefore appear at approximately the same average power.

Numerical and experimental results of structure B show that if the device is used at the cavity resonance wavelength, then at high incident average power, the thermal effects will cause a significant decrease of the switching contrast $C$ because of a mismatch between the incident signal wavelength and $\lambda_{\text {res }}$. Furthermore, it is seen that $C$ depends on the signal FF, i.e., it is higher at lower FF, owing to a higher peak power.

2) Similar measurements were performed on the structure $A$ device, which has better thermal properties. As expected, a much higher switching contrast was obtained with this device, since pump signals of higher average power (more than $16 \mathrm{dBm}$ ) could be used before observing the increase of $R_{\text {off }}$. However, because of limited device availability, this device had a faster response time (1.5 ps) than the structure B device (3 ps). As a result [see (11)], a higher peak power is needed to achieve a given carrier density and, thus, a given amount of absorption saturation. So, in order to minimize the negative effects of heating at high power and demonstrate a large switching contrast with this high-speed device, we chose to operate the device on the long wavelength side of the microcavity resonance. In this configuration, resonance matching could be achieved at a higher incident power, owing to the shift of the cavity resonance to a longer wavelength. As the resonance wavelength for this structure is equal to $1557 \mathrm{~nm}$, the pump wavelength was set to $1554 \mathrm{~nm}$ and the probe wavelength to $1560.5 \mathrm{~nm}$. Fig. 6(a) and (b) presents the switching contrast and the reflectance values $R_{\text {on }}$ and $R_{\text {off }}$ as a function of average incident power $P_{\text {ave }}$. Numerical calculation has also been done, and the parameter values used for this simulation are indicated in Table I. As noted previously, a very good agreement is observed between the numerical and the experimental results. 


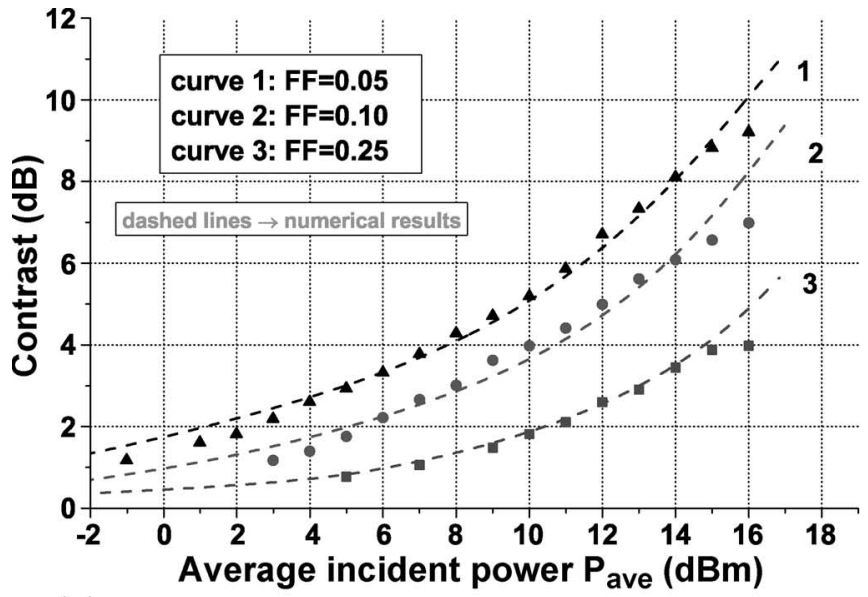

(a)

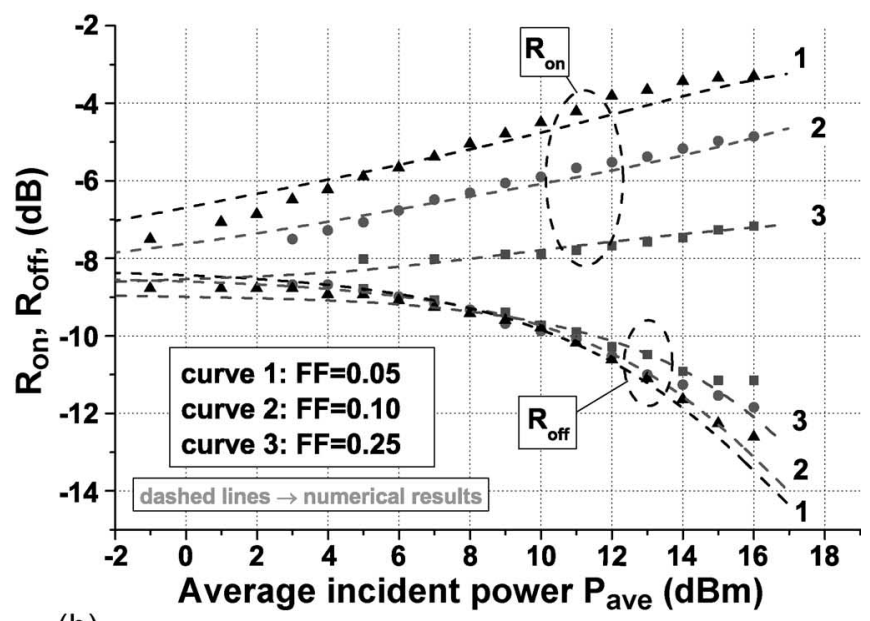

(b)

Fig. 6. Experimental (symbols) and numerical (full curve) results from structure A for (a) switching contrast $C$ and (b) device reflectances $R_{\text {on }}$ and $R_{\text {off }}$ versus average incident power $P_{\text {ave }}$ obtained for three values of $\mathrm{FF}(0.25,0.1$, and 0.05). Numerical calculation and experiment are done with an initial active layer temperature $T_{\text {act }}$ of $300 \mathrm{~K}$ and with $\lambda_{\text {work }} 3.5 \mathrm{~nm}$ higher than $\lambda_{\text {res }}$ at low incident average power. (a) Average incident power $P_{\text {ave }}$ (in decibel meters). (b) Average incident power $P_{\text {ave }}$ (in decibel meters).

As expected, it is seen that $R_{\text {on }}$ increases with average optical power and with peak power owing to the saturation of the absorption [cf. Fig. 6(b)], but this time, $R_{\text {off }}$ decreases with incident average power, up to the highest value available in the experiment, i.e., $16 \mathrm{dBm}$. This is also owing to the heating effects of the optical pump that cause both the cavity and exciton resonance red shifts, but as the probe wavelength was set to $3.5 \mathrm{~nm}$ higher than $\lambda_{\text {res }}$, the cavity resonance comes closer to the probe wavelength, and as a result, $R_{\text {off }}$ decreases. Consequently, the switching contrast keeps increasing with incident power of up to $16 \mathrm{dBm}$ [cf. Fig. 6(a)]. These results show that a device with good thermal properties leads to improved switching characteristics at high power. Furthermore, an optimized switching contrast is obtained when the SA microcavity is operated at a longer wavelength than the low intensity resonance in order to partially compensate the negative effects of optically induced heating. Of course, this strategy can only work up to a maximum value of average incident power, and the numerical results show that for structure A, the switching contrast starts decreasing at an average power larger than $17 \mathrm{dBm}$.

\section{Discussion}

With relaxation times down to $1.5 \mathrm{ps}$, SA-based devices are fast enough to be compatible with processing at $160 \mathrm{~Gb} / \mathrm{s}$ [24], so it can be expected that the device performance will essentially be bit rate independent up to this rate. The other limiting factor in device performance is that of thermal effects. However, it has been demonstrated in this paper that with a good thermal management, it is possible to have a contrast of $4 \mathrm{~dB}$ with an FF of 0.25 , despite the thermal effects. This thermal management consists of two key points.

The first key point is to design a device with an effective thermal resistance as low as possible in order to limit the active layer temperature rise. In this paper, the use of a broadband metallic (silver) back mirror with an indium-gold bonding layer has proved to be a convenient solution to having efficient thermal dissipation. As the resonance wavelength shift is proportional to the active layer temperature, it is 5.5 times higher for structure B than for structure A ( $0.54 \mathrm{~nm}$ compared to $0.08 \mathrm{~nm} / \mathrm{mW}$ of absorbed power); hence, for structure $\mathrm{B}$, we have an earlier and quicker contrast variation with the average power than with structure A [cf. Figs. 5(a) and 6(a)].

The second key point is to operate the device to anticipate the negative thermal effects. The solution proposed in this paper is to introduce a wavelength offset between the cavity resonance and the optical signal. In this way, resonance matching is reached with higher values of average power to maximize the switching contrast [cf. Fig. 6(a) and (b)].

From the results obtained in this study, we expect that the use of a low thermal resistance device with an appropriate detuning between the signal wavelength and the cavity resonance will allow the realization of $2 \mathrm{R}$ regeneration of $160-\mathrm{Gb} / \mathrm{s}$ signals. Indeed, an RZ 50\% duty cycle optical signal has an FF of 0.25, and under this condition, the results in Fig. 6(a) show that a 4- $\mathrm{dB}$ extinction ratio improvement can be obtained, which is adequate for $2 \mathrm{R}$ regeneration [22]. Further improvement could be obtained by reducing even further the effective thermal resistance, as, for instance, by having a top-down mounting with copper-copper direct bonding [41] onto a silicon carbide wafer or even onto a block of copper. It could also be interesting to use an impedance-matched microcavity with a very small number of quantum wells (typically one to three QWs) combined with a low-loss broadband metal-based back mirror [42], [43], which could give low insertion loss with a high switching contrast at sufficiently low average power to avoid thermal effects.

\section{CONCLUSion}

In summary, the optical and thermal characteristics of quantum-well microcavity SA switching gates have been presented. Calculations, which take into account Fabry-Perot microcavity, quantum-well saturable absorption, bottom mirror 
nonsaturable loss, and a phenomenological description of the resonance shift with carrier density and the active material temperature, have been carried out with a self-consistent method. The numerical results are largely confirmed by experiments in which the FF of the input signal has been varied. Numerical and experimental results show that the thermal effects have to be taken into account to enhance the extinction ratio at high pulse repetition rates. With a device speed and a signal FF compatible with a bit rate of $160 \mathrm{~Gb} / \mathrm{s}$, both calculations and experiments show that improved SA devices should be capable of handling $2 \mathrm{R}$ regeneration, with a sufficiently large switching contrast.

\section{ACKNOWLEDGMENT}

The authors would like to thank H. Bernas of CSNSM, Orsay, France, for the ion irradiation and J. Even of FOTON, Rennes, France, for the numerical data based on the equation of Bethe-Salpeter.

\section{REFERENCES}

[1] O. Leclerc, "Optical versus electronic in-line signal processing in optical communication systems: An exciting challenge for optical devices," in Proc. ECIO, 2003, pp. 55-67.

[2] L. Lucek and K. Smith, "All-optical signal regenerator," Opt. Lett., vol. 18 , no. 15 , pp. 1226-1228, Aug. 1993.

[3] K. E. Stubkjaer, "Semiconductor optical amplifier-based all-optical gates for high-speed optical processing," IEEE J. Sel. Topics Quantum Electron., vol. 6, no. 6, pp. 1428-1435, Nov./Dec. 2000.

[4] P. S. Cho, D. Mahgerefteh, and J. Goldhar, "All-optical 2R regeneration and wavelength conversion at $20 \mathrm{~Gb} / \mathrm{s}$ using an electroabsorption modulator," IEEE Photon. Technol. Lett., vol. 11, no. 12, pp. 1662-1664, Dec. 1999.

[5] D. Atkinson, W. H. Loh, V. V. Afanasjev, A. B. Grudinin, A. J. Seeds, and D. N. Payne, "Increased amplifier spacing in a soliton system with quantum-well saturable absorbers and spectral filtering," Opt. Lett., vol. 19 , no. 19 , pp. 1514-1516, Oct. 1994.

[6] K. Nonaka, Y. Noguchi, H. Tsuda, and T. Kurokawa, "Digital signal regeneration with side-injection-light-controlled bistable laser diode as a wavelength converter," IEEE Photon. Technol. Lett., vol. 7, no. 1, pp. 29-31, Jan. 1995.

[7] J. Mangeney, G. Aubin, L. Oudar, J. C. Harmand, G. Patriarche, H. Choumane, N. Stelmakh, and J. M. Lourtioz, "All-optical discrimination at $1.55 \mu \mathrm{m}$ using ultrafast saturable absorber vertical cavity device," Electron. Lett., vol. 36, no. 17, pp. 1486-1488, Aug. 2000.

[8] A. Hirano, H. T. Tsuda, K. Hagimoto, R. Takahashi, Y. Kawamura, and H. Iwamura, "10 ps pulse all-optical discrimination using a highspeed saturable absorber optical gate," Electron. Lett., vol. 31, no. 9, pp. 736-737, Apr. 1995

[9] C. Porzi, A. Isomäki, M. Guina, and O. G. Okhotnikov, "Impedancedetuned high-contrast vertical cavity semiconductor switch," in Proc. $O F C$, Anaheim, CA, 2005, p. 3

[10] E. Burr, M. Pantouvaki, M. Fice, R. Gwilliam, A. Krysa, J. Roberts, and A. Seeds, "Signal stability in periodically amplified fiber transmission systems using multiple quantum well saturable absorbers for regenerations," J. Lightw. Technol., vol. 24, no. 2, pp. 747-754, Feb. 2006

[11] O. G. Okhotnikov, T. Jouhti, J. Konttinen, S. Karirinne, and M. Pessa, "1.5 $\mu \mathrm{m}$ monolithic GaInNAs semiconductor saturable-absorber mode locking of an erbium fiber laser," Opt. Lett., vol. 28, no. 5, pp. 364-366, Mar. 2002.

[12] Y. Sakakibara, A. G. Rozhin, H. Kataura, Y. Achiba, and M. Tokumoto, "Carbon nanotube-poly(vinylalcohol) nanocomposite film devices: Applications for femtosecond fiber laser mode lockers and optical amplifier noise suppressors," Jpn. J. Appl. Phys., vol. 44, no. 4A, pp. 1621-1625, 2005.

[13] J. C. Simon, L. Bramerie, F. Ginovart, V. Roncin, M. Gay, S. Feve, C. E. Le, and M. L. Chares, "All-optical regeneration techniques," Ann. Telecommun., vol. 58, no. 11/12, pp. 1708-1724, 2003.
[14] D. Massoubre, J. L. Oudar, J. Dion, J. C. Harmand, A. Shen, J. Landreau, and J. Decobert, "Scaling of the switching energy in microcavity saturable absorber devices," Appl. Phys. Lett., vol. 88, no. 10, p. 153513, Apr. 2006.

[15] R. Takahashi, Y. Kawamura, T. Kagawa, and H. Iwamura, "Ultrafast $1.55 \mu \mathrm{m}$ photoresponses in low-temperature-grown InGaAs/InAlAs quantum wells," Appl. Phys. Lett., vol. 65, no. 14, pp. 1790-1792, Oct. 1994.

[16] P. W. Smith, Y. Silberger, and D. A. B. Miller, "Mode locking of semiconductor diode lasers using saturable excitonic nonlinearities," J. Opt. Soc. Amer. B, Opt. Phys., vol. 2, no. 7, pp. 1228-1236, Jul. 1985.

[17] E. L. Delpon, J. L. Oudar, N. Bouche, R. Raj, A. Shen, N. Stelmakh, and J. M. Lourtioz, "Ultrafast excitonic saturable absorption in ion-implanted InGaAs/InAlAs multiple quantum wells," Appl. Phys. Lett., vol. 72, no. 7, pp. 759-761, Feb. 1998.

[18] L. Qian, S. D. Benjamin, P. W. E. Smith, B. J. Robinson, and D. A. Thompson, "Subpicosecond carrier lifetime in beryllium-doped InGaAsP grown by He-plasma-assisted molecular beam epitaxy," Appl. Phys. Lett., vol. 71, no. 11, pp. 1513-1515, Sep. 1997.

[19] M. Guézo, S. Loualiche, J. Even, A. L. Corre, O. Dehaese, Y. Pellan, and A. Marceaux, "Nonlinear absorption temporal dynamics of Fe-doped GaInAs/InP multiple quantum wells," J. Appl. Phys., vol. 94, no. 4, pp. 2355-2359, Aug. 2003

[20] J. Mangeney, N. Stelmakh, A. Shen, J.-M. Lourtioz, A. Alexandrou, J.-P. Likforman, C. Clerc, V. Thierry-Mieg, E. Lugagne-Delpon, and J.-L. Oudar, "Sub-picosecond wideband efficient saturable absorber created by high energy $(200 \mathrm{MeV})$ irradiation of $\mathrm{Au}^{+}$ions into bulk GaAs," Electron. Lett., vol. 34, no. 8, pp. 818-820, Apr. 1998.

[21] M. Gicquel-Guézo, S. Loualiche, J. Even, C. Labbé, O. Dehaese, A. L. Corre, H. Folliot, and Y. Pellan, "290 fs switching time of Fe-doped quantum well saturable absorbers in a microcavity in $1.55 \mu \mathrm{m}$ range," Appl. Phys. Lett., vol. 85, no. 24, pp. 5926-5928, Dec. 2004.

[22] D. Rouvillain, P. Brindel, F. Seguineau, L. Pierre, O. Leclerc, H. Choumane, G. Aubin, and J. L. Oudar, "Optical 2R regenerator based on passive saturable absorber for $40 \mathrm{~Gb} / \mathrm{s}$ WDM long-haul transmissions," Electron. Lett., vol. 38, no. 19, p. 1113, 2002.

[23] M. Gay, L. Bramerie, D. Massoubre, A. O'Hare, A. Shen, J. L. Oudar, and J. C. Simon, "Cascadability assessment of a $2 \mathrm{R}$ regenerator based on saturable absorber and semiconductor optical amplifier in a path switchable recirculating loop," IEEE Photon. Technol. Lett., to be published.

[24] D. Massoubre, J. Fatome, S. Pitois, J. Decobert, J. Landreau, G. Millot, and J. L. Oudar"All-optical extinction ratio enhancement of a $160 \mathrm{GHz}$ pulse train using a saturable absorber vertical microcavity," Opt. Lett., vol. 31, no. 4, pp. 537-539, Feb. 2006.

[25] O. Krebs and P. Voisin, "Giant optical anisotropy of semiconductor heterostructures with no common atom and the quantum-confined Pockels effect," Phys. Rev. Lett., vol. 77, no. 9, pp. 1829-1832, Aug. 1996.

[26] O. Krebs, D. Rondi, J. L. Gentner, L. Goldstein, and P. Voisin1, "Inversion asymmetry in heterostructures of zinc-blende semiconductors: Interface and external potential versus bulk effects," Phys. Rev. Lett., vol. 80, no. 26, pp. 5770-5773, Jun. 1998.

[27] E. L. Cren, S. Lobo, S. Féve, and J. C. Simon, "Polarization sensitivity characterization under normal incidence of a multiple quantum wells saturable absorber nonlinear mirror as a function of the temperature of the chip," Opt. Commun., vol. 254, no. 1-3, pp. 96-103, 2005.

[28] C. C. Lee, C. Y. Wang, and G. Matijasevic, "Au-In bonding below the eutectic temperature," IEEE Trans. Compon., Hybrids, Manuf. Technol., vol. 16, no. 3, pp. 311-316, May 1993

[29] M. Born and E. Wolf, Principles of Optics, 6th ed. New York: Pergamon, 1980, pp. 325-326

[30] S. Corzine, R. S. Geels, J. Scott, R. Yan, and L. A. Coldren, "Design of Fabry-Perot surface-emitting lasers with a periodic gain structure," IEEE J. Quantum Electron., vol. 25, no. 6, pp. 1513-1524, Jun. 1989.

[31] H. Haug and S. Schmitt-Rink, "Basic mechanisms of the optical nonlinearities of semiconductors near the band edge," J. Opt. Soc. Amer. B, Opt. Phys., vol. 2, no. 7, pp. 1135-1142, Jul. 1985.

[32] D. Campi, P. J. Bradley, R. Calvani, and R. Caponi, "Modeling of nonlinear absorption and refraction in quantum-well structures for all-optical switching," IEEE J. Quantum Electron., vol. 29, no. 4, pp. 1144-1157, Apr. 1993.

[33] S. H. Park, J. F. Morhange, A. D. Jeffery, R. A. Morgan, A. ChavezPirson, H. M. Gibbs, S. W. Koch, N. Peyghambarian, M. Derstine, A. C. Gossard, J. H. English, and W. Weigmann, "Measurements of roomtemperature band-gap-resonant optical nonlinearities of GaAs/AlGaAs multiple quantum wells and bulk GaAs," Appl. Phys. Lett., vol. 52, no. 15, pp. 1201-1203, Apr. 1988 
[34] A. M. Fox, A. C. Maciel, M. G. Shorthose, J. F. Ryan, M. D. Scott, J. L. Davies, and J. R. Riffat, "Nonlinear excitonic optical absorption in GaInAs/InP quantum wells," Appl. Phys. Lett., vol. 51, no. 1, pp. 30-32, Jul. 1987.

[35] Y. P. Varshni, "Temperature dependence of the energy gap in semiconductors," Physica, vol. 34, no. 1, p. 149, 1967.

[36] D. K. Gaskill, N. Bottka, L. Aina, and M. Mattingly, "Band-gap determination by photoreflectance of InGaAs and InAlAs lattice matched to InP," Appl. Phys. Lett., vol. 56, no. 13, pp. 1269-1271, 1990.

[37] E. Zielinski, H. Schweizer, K. Streubel, H. Eisele, and G. Weimann, "Excitonic transitions and exciton damping processes in InGaAs/InP," J. Appl. Phys., vol. 59, no. 6, pp. 2196-2204, 1986.

[38] M. Kondow, T. Kitatani, K. Nakahara, and T. Tanaka, "Temperature dependence of lasing wavelength in a GaInNAs laser diode," IEEE Photon. Technol. Lett., vol. 12, no. 7, pp. 777-779, Jul. 2000.

[39] S. Rapp, J. Piprek, K. Streubel, J. André, and J. Wallin, "Temperature sensitivity of $1.54-\mu \mathrm{m}$ vertical-cavity lasers with an InP-based Bragg reflector," J. Appl. Phys., vol. 33, no. 10, pp. 1839-1845, 1997.

[40] J. Mangeney, J. Lopez, N. Stelmakh, J. M. Lourtioz, J. L. Oudar, and H. Bernas, "Subgap optical absorption and recombination center efficiency in bulk GaAs irradiated by light or heavy ions," Appl. Phys. Lett., vol. 76, no. 1, pp. 40-42, Jan. 2000.

[41] T. H. Kim, M. M. R. Howlader, T. Itoh, and T. Suga, "Room temperature $\mathrm{Cu}-\mathrm{Cu}$ direct bonding using surface activated bonding method," J. Vac. Sci. Technol. A, Vac. Surf. Films, vol. 21, no. 2, pp. 449-453, 2003.

[42] C. Symonds, J. Dion, I. Sagnes, M. Dainese, M. Strassner, L. Leroy, and J.-L. Oudar, "High performance $1.55 \mu \mathrm{m}$ vertical external cavity surface emitting laser with broadband integrated dielectric-metal mirror," Electron. Lett., vol. 40, no. 12, pp. 734-735, Jun. 2004.

[43] T. Nguyen, C. Shen, X. Wu, T. Pinnington, J. Krogen, B. Witzigmann, C. Tsai, L. Cote, M. Geva, D. Huynh, A. Konkar, and P. C. Chen, "Design and fabrication of $1.3-\mu \mathrm{m}$ vertical-cavity surface-emitting lasers using dielectric reflectors," IEEE Photon. Technol. Lett., vol. 15, no. 11, pp. 1498-1500, Nov. 2003.

David Massoubre was born in France in 1979. He received the degree in physics engineering from the National Institute of Applied Sciences, Rennes, France, in 2002. He is currently working toward the Ph.D. degree at the Laboratory for Photonics and Nanostructures, Centre National de la Recherche Scientifique, Marcoussis, France. His Ph.D. thesis is focused on all-optical saturable absorber-based microcavities for passive optical signal regeneration at ultrahigh bit rate.
Jean-Louis Oudar received the degree from Ecole Polytechnique, Paris, France, in 1971 and the Doctorat es-Sciences degree in physics from the University of Paris in 1977.

Since joining the Centre National d'Etudes des Telecommunications (CNET) in 1971, he has worked on nonlinear optical phenomena in condensed matter. Studying the enhanced optical nonlinearities of organic compounds, he developed the basis of a molecular engineering approach for nonlinear organic materials. In 1979, he was a Visiting Scientist at the Physics Department, University of California at Berkeley, where he worked on new techniques of nonlinear spectroscopy based on four-wave mixing. In 1980, he returned to CNET, which later evolved as France Telecom R\&D. There, he successively led the Laboratory for Optics Physics and Materials and the Laboratory of Quantum Optoelectronic Devices. His research has been conducted on the ultrafast dynamic nonlinearities of III-V semiconductor microstructures, quantum optics of microcavities, and the development of optical bistable and switching devices. From 1991 to 2003, he was a Lecturer in physics at Ecole Polytechnique. In 2000, he joined the newly created Laboratory for Photonics and Nanostructures of the Centre National de la Recherche Scientifique (CNRS-LPN), Marcoussis, France, where he is currently leading the Photonic Devices for Telecommunications Group. He has authored or coauthored more than 150 research papers in refereed journals, presented more than 60 invited talks at international conferences, and holds nine patents in the field of laser and nonlinear optical devices.

Dr. Oudar has been active in the editorial board of several scientific journals and currently with the European Physical Journal D.

Photographs and biographies of Arthur O'Hare, Mathilde Gay, Laurent Bramerie, Jean-Claude Simon, Alexandre Shen, and Jean Decobert not available at time of publication. 